\title{
Unraveling Affective Dysregulation in Borderline Personality Disorder: A Theoretical Model and Empirical Evidence
}

\author{
Ulrich W. Ebner-Priemer \\ Karlsruhe Institute of Technology, \\ Germany and Central Institute of Mental Health Mannheim, \\ Germany \\ Philip Santangelo \\ Karlsruhe Institute of Technology, Germany \\ Francis Tuerlinckx \\ KU Leuven, Belgium \\ Gregory Verleysen and Katrijn Van Deun
KU Leuven, Belgium
}

\author{
Marlies Houben
KU Leuven, Belgium \\ Marlies Houben
KU Leuven, Belgium
}

Nikolaus Kleindienst

Central Institute of Mental Health Mannheim, Germany

Zita Oravecz

University of California-Irvine

Martin Bohus

Central Institute of Mental Health Mannheim, Germany

\author{
Peter Kuppens \\ KU Leuven, Belgium
}

\begin{abstract}
Although emotion dysregulation has consistently been conceptualized as a core problem of borderline personality disorder (BPD), a comprehensive, and empirically and ecologically validated model that captures the exact types of dysregulation remains absent. In the present article, we combine insights from basic affective science and the biosocial theory of BPD to present a theoretical model that captures the most fundamental affective dynamical processes that underlie BPD and stipulates that individuals with BPD are characterized by more negative affective homebases, higher levels of affective variability, and lower levels of attractor strength or return to baseline. Next, we empirically validate this proposal by statistically modeling data from three electronic diary studies on emotional responses to personally relevant stimuli in personally relevant environments that were collected both from patients with $\operatorname{BPD}(N=50,42$, and 43) and from healthy subjects $(N=50,24$, and 28). The results regarding negative affective homebases and heightened affective variabilities consistently confirmed our hypotheses across all three datasets. The findings regarding attractor strengths (i.e., return to baseline) were less consistent and of smaller magnitude. The transdiagnostic nature of our approach may help to elucidate the common and distinctive mechanisms that underlie several different disorders that are characterized by affective dysregulation.
\end{abstract}

Although emotion dysregulation has been consistently conceptualized as the core problem of borderline personality disorder (BPD; Crowell et al., 2008; Lieb, Zanarini, Schmahl, Linehan, \& Bohus, 2004; Linehan, 1993; Links et al., 2007; Siever, Torgersen,
Gunderson, Livesley, \& Kendler, 2002; Skodol et al., 2002a, 2002b), a comprehensive and empirically validated account that captures the exact types of dysregulation that people suffering from BPD encounter in their daily lives remains absent. In the
This article was published Online First January 19, 2015.

Ulrich W. Ebner-Priemer, Karlsruhe Institute of Technology, Karlsruhe, Germany and Department of Psychiatry and Psychotherapy, Central Institute of Mental Health Mannheim, Germany; Marlies Houben, Faculty of Psychology and Educational Sciences, KU Leuven, Leuven, Belgium; Philip Santangelo, Karlsruhe Institute of Technology, Karlsruhe, Germany; Nikolaus Kleindienst, Department of Psychiatry and Psychotherapy, Central Institute of Mental Health Mannheim, Germany; Francis Tuerlinckx, Faculty of Psychology and Educational Sciences, KU Leuven, Belgium; Zita Oravecz, Department of Cognitive Sciences, University of California-Irvine; Gregory Verleysen and Katrijn Van Deun, Faculty of Psychology and Educational Sciences, KU Leuven, Belgium; Martin Bohus, Department of Psychiatry and Psychotherapy, Central Institute of Mental Health Mannheim, Germany; Peter Kuppens, Faculty of Psychology and Educational Sciences, KU Leuven, Belgium.
Zita Oravecz is now at the Department of Human Development and Family Studies, The Pennsylvania State University.

The authors have no conflicts of interest to declare. The research was supported in part by the Research Fund of KU Leuven (GOA/15/003 and OT/11/31), by the Interuniversity Attraction Poles program financed by the Belgian government (IAP/P7/06), and by the Fund for Scientific Research-Flanders (FWO). Ulrich W. Ebner-Priemer and Marlies Houben contributed equally to this work.

Correspondence concerning this article should be addressed to Ulrich W. Ebner-Priemer, Chair of Applied Psychology, Karlsruhe Institute of Technology, Engler-Bunte-Ring 15, Geb. 40.40, D-76131 Karlsruhe, Germany. E-mail: ulrich.ebner-priemer@kit.edu 
present paper, we first review findings from studies of emotion dysregulation in BPD in the laboratory and in everyday life. We next formulate the criteria for a comprehensive framework that captures affective dysregulation in BPD and emphasize the need for theoretical models that are rooted in basic affective science and for appropriate data collection strategies and associated statistical modeling tools. Next, we propose such a theoretical model (the DynAffect model, which proposes that individual differences in core affect patterns can be captured in terms of three fundamental processes: affective homebase, affective variability, and attractor strength) and apply this framework to several independent datasets that involve symptomlike basic affective processes in daily life. With this approach, we aim to take advantage of the progress in basic affective science to enable comparisons of mechanisms and processes across studies and across disorders.

\section{Studies on Emotion Dysregulation in BPD}

Studies that have examined emotion dysregulation in BPD in the laboratory have not always produced consistent results concerning the exact nature of emotion dysregulation in BPD. Studies that have utilized the startle response paradigm have yielded mixed findings; some studies have shown heightened reactivity in BPD patients (Donegan et al., 2003; Ebner-Priemer et al., 2005), whereas others have not or have even found hyporeactivity (Herpertz, Kunert, Schwenger, \& Sass, 1999; Herpertz et al., 2001). The findings of some studies were dependent on the stimuli and revealed effects for BPD-specific stimuli but not for neutral stimuli (Hazlett et al., 2007; Limberg, Barnow, Freyberger, \& Hamm, 2011) or effects of specific emotions such as fear but not anger (Minzenberg, Fan, New, Tang, \& Siever, 2007). Additionally, a dissociation between physiological measures and subjective reports has been reported (Hazlett et al., 2007; Herpertz et al., 2001; Koenigsberg et al., 2009; Limberg et al., 2011). The findings of psychophysiological studies that have used standardized film clips are also mixed; some of these studies have reported higher baseline values and unchanged reactivity (Kuo \& Linehan, 2009), whereas other studies have reported the opposite pattern (Austin, Riniolo, $\&$ Porges, 2007). Other studies have examined patients on medication (Elices et al., 2012; Staebler, Gebhard, Barnett, \& Renneberg, 2009), which makes the interpretation of the results difficult. Studies that have used standardized laboratory stressors have revealed heightened baseline values (Jacob et al., 2009; Reitz et al., 2012), no differences in reactivity (Jacob et al., 2009; Reitz et al., 2012; Schmahl et al., 2004), and a slower return to baseline in patients with BPD (Reitz et al., 2012). However, these results are also strongly dependent on the outcome measures; for example, different patterns have been reported for negative affect and shame (Gratz, Rosenthal, Tull, Lejuez, \& Gunderson, 2010). In contrast, the findings of studies that have used e-diaries (Nica \& Links, 2009; Santangelo, Bohus, \& Ebner-Priemer, 2014) are more conclusive than those of the laboratory studies. Compared to healthy controls, heightened intensities of emotions and physiological parameters (Ebner-Priemer et al., 2007b), heightened instability of emotions (Ebner-Priemer et al., 2007a; Trull et al., 2008), higher affective reactivity during social interactions (Sadikaj, Moskowitz, Russell, Zuroff, \& Paris, 2013), and prolonged persistence of emotions have been consistently shown (Reisch, Ebner-Priemer, Tschacher, Bohus, \& Linehan, 2008; Stiglmayr et al., 2005).
However, findings regarding specificity have been disappointing. Santangelo et al. (2014) assessed affective instability in patients with BPD, posttraumatic stress disorder, and bulimia nervosa using e-diaries. They were not able to differentiate groups according to global instability indices, that is, instability did not show sufficient specificity. Therefore, Santangelo et al. (2014) argue that analyzing multiple subcomponents of the dynamic processes (e.g., sensitivity, reactivity, and slow return to baseline) might be necessary to delineate existing differences of the emotional processes between patients with BPD and clinical controls.

\section{Criteria for a Framework for the Investigation of Affective Dysregulation}

Based on the above review of the literature, it is difficult to draw firm conclusions regarding exactly how emotion dysregulation in BPD should be understood. Moreover, the existing research on emotion dysregulation in BPD suffers from one or more of the following shortcomings.

First, there is neither a consistent definition nor an integrative framework that describes the affective experience of BPD. Accordingly, different emotional components are operationalized by identical parameters, and identical emotional components are operationalized by different parameters. In a review of the components of emotion dysregulation in BPD, Carpenter and Trull (2013) stated, "This practice . . . threatens to render the term "emotion dysregulation' vague and perhaps meaningless (p. 335)." Clearly, we are missing an overarching theoretical model and clear definitions from basic (affective) science. Such an overarching model would also enable the comparison of mechanisms and processes across studies and across patients with different disorders.

Second, most studies have assessed emotions as timely fixed states and do not examine the time-varying nature of emotions. For example, during the presentation of affective pictures, it is assumed that people are in a constant and unchanging emotional state, and the unfolding of emotions across time is not taken into account. However, a fundamental characteristic of emotions is that they continuously change over time. Affective ups and downs inform people about important events that present threats or opportunities and allow them to respond with appropriate actions (Kuppens, Oravecz, \& Tuerlinckx, 2010). Even some of the core symptoms of BPD have been formulated in dynamical terms, such as the classification criterion for "affective instability." Therefore, studies that allow for the assessment of the dynamical aspects of emotions in BPD (via the examination of multiple assessment points over time and the utilization of statistical models that are capable of tracking dynamics, mechanisms, and processes) are needed.

Third, most studies have investigated single components of affective dysregulation, such as hyperreactivity. However, the components of emotion regulation and the psychopathological symptoms are parts of an integrative whole. For example, Linehan's biosocial theory of BPD (Linehan, 1993) defines three interdependent components of emotion dysregulation: (a) high sensitivity to emotional stimuli, which translates into a lower threshold for responding to or recognizing (negative) emotional stimuli, (b) intense responses to emotional stimuli, which is reflected by emotional responses with greater amplitudes, and (c) slow return to baseline, which translates into longer duration of 
emotional responses (Crowell et al., 2008; Linehan, 1993). There is a clear lack of studies that simultaneously analyze multiple components to create full accounts of emotion dysregulation in BPD and account for possible theoretical and statistical overlap in a manner similar to the formulation of Linehan's theoretical framework (Carpenter \& Trull, 2013; Sadikaj, Russell, Moskowitz, \& Paris, 2010). Clearly, simultaneous assessments and analyses of all the relevant components that are guided by an integrative theoretical framework and associated statistical models are needed.

Fourth, Elices et al. (2012), Jacob et al. (2009), Kuo and Linehan (2009), and Rosenthal et al. (2008) hypothesized that the mixed and inconclusive findings from laboratory studies might, at least partially, be explained by the use of stimuli that are not personally relevant in situations/environments (i.e., the laboratory) that are not personally relevant. In their everyday lives, patients are not faced with single stimuli that are separated by intertrial intervals; rather, patients experience a wealth of varied and quickly changing emotional stimuli. Accordingly, Rosenthal et al. (2008) argued that stimuli of greater personal and ecological relevance are needed for future research.

An overarching theoretical model of emotional dynamics that involves multiple components of dysregulation, an assessment method to gather data about people's emotional responses to multiple personally relevant stimuli (to capture the dynamical processes) in personally relevant environments, and a statistical model that is capable of analyzing the resulting data are needed to address these shortcomings. Fortunately, solutions for all three of these problems have been developed in recent years. In the following section, we will propose a theoretical model of emotional dynamics (the DynAffect Model), a methodological approach to assess the affective dynamics in response to personally relevant stimuli in personally relevant environments (ambulatory assessment), and a statistical framework that enables the application of the theoretical model to the empirical data (multilevel modeling approaches).

\section{Components of a Comprehensive Framework for the Investigation of Affective Dysregulation}

\section{The DynAffect Model}

The DynAffect model was originally conceived as a theoretical account of the dynamics of affect in the field of basic affective sciences. The aim of this model was to synthesize different lines of basic research on emotion dynamics into one coherent model that identifies the major processes that underlie the general population's individual differences in the temporal dynamics of affective experiences, and use it to generate new predictions about emotion dynamics in both the normal and abnormal range of variation (Kuppens, Oravecz, \& Tuerlinckx, 2010).

In the DynAffect model, affective experiences are described in terms of core affect. As defined by Russell (2003), core affect reflects an integral blend of the two fundamental dimensions of affect, valence and arousal, that characterizes subjective feelings. More specifically, at each point in time, a person's feeling state can be characterized in terms of how good or bad (valence) and how active or passive (arousal) he or she is feeling. Core affect continuously changes over time as a result of internal and external events and regulation efforts, and individuals differ in their typical patterns of such changes. According to the DynAffect model, individual differences in core affect patterns can be captured in terms of three fundamental processes: (a) an affective homebase, which is a baseline attractor state around which affect fluctuates. It reflects the average or normative affective state of a person and lies at the center of the affective fluctuations across time and contexts. A more positive or negative homebase signifies that an individual will experience more positive or negative emotions. To be clear, the attractor state does not necessarily reflect the state that an individual may intentionally prefer, but rather the state to which affect is drawn back to when deviating from it because of regulatory processes and habits characterizing the individual; (b) a level of affective variability, which reflects the affective deviations of or fluctuations around the homebase that result from how the individual responds to both internal or external processes or events. High levels of variability reflect affective trajectories that show stronger deviations from the homebase, and vice versa; and (c) attractor strength, which refers to a regulatory force that pulls deviating affective fluctuations back toward the homebase and enables emotional recovery. Attractor strength is thought to reflect regulatory and homeostatic processes (for more details, see Kuppens, Oravecz, \& Tuerlinckx, 2010): The higher an individual's attractor strength, the more strongly deviations from the homebase are pulled back toward the affective homebase.

There is emerging and consolidating empirical evidence that differences in these three dynamical properties of affective homebase, affective variability, and attractor strength of the DynAffect are, to a large extent, capable of explaining the individual differences in temporal patterns and trajectories that have been observed in people's affective experiences. For a more detailed description of the DynAffect theory, please see Kuppens, Oravecz, and Tuerlinckx (2010). Moreover, the basic tenets of DynAffect can be used to generate novel predictions regarding affect dynamics, in both clinical populations, such as, for instance, BPD, as well as in the normal population (e.g., that the attractor strength increases with increasing distance from the homebase).

The fundamental processes proposed by the DynAffect theory can be mapped onto the elements of the biosocial theory of BPD proposed by Linehan (1993). First, the proposed high sensitivity to emotional stimuli influences the affective homebase. Heightened sensitivity to negative emotional stimuli has been described for BPD (Daros, Zakzanis, \& Ruocco, 2013; Domes, Schulze, \& Herpertz, 2009; Matzke, Herpertz, Berger, Fleischer, \& Domes, 2014), which means that persons with BPD are more likely to respond to or recognize these types of stimuli. This heightened sensitivity would lead to a tendency to experience more negative emotions. Therefore, we hypothesize that BPD patients will exhibit a more negative baseline, without implying that individuals with BPD prefer more negative states, but rather that their affect is automatically drawn back to a more negative attractor state. Second, the intense responses to emotional stimuli result in increased emotional reactivity to these stimuli, which leads to larger fluctuations around the homebase state. This would be captured by higher levels of affective variability in the model. Third, reduced attractor strength can be regarded as reflective of a slow return to baseline. A reduced attractor strength would mean that if BPD patients emotionally react to an internal or external event, and consequently deviate from their homebase state, they experience 
less "pull back" strength toward their homebase state; as a consequence, they tend to stay longer in that aberrant state (and hence have a slower return to baseline). Consequently, the biosocial theory of Linehan (1993) can be regarded as a specific instantiation of the different dynamic processes comprising the DynAffect model. To test this model in BPD in an ecologically valid manner, data that capture the dynamics of affective experiences in the course of everyday life are needed.

\section{Ambulatory Assessment}

Ambulatory assessment covers a wide range of assessment methods that are used to study people in their natural environments, including computerized methods of experience sampling (e-diaries) and continuous psychophysiological, biological, and behavior monitoring. The main characteristics are: (a) real time assessments that minimize retrospective bias; (b) repeated assessments that enable the modeling of dynamic processes; and (c) assessments that occur in real life situations to ensure that affective responses to personally relevant stimuli in personally relevant environments are assessed (Trull \& Ebner-Priemer, 2013). Ambulatory assessment is a particularly well-suited method for assessing the dynamics of psychopathological symptoms as it captures the film of life rather than a snapshot (Ebner-Priemer, Eid, Kleindienst, Stabenow, \& Trull, 2009). However, please note that ambulatory assessment and laboratory experiments are not fundamentally opposed alternatives, but instead should be considered as complementary approaches (Ebner-Priemer \& Trull, 2009).

\section{Statistical Models}

To make the DynAffect model amenable to the empirical study of such data, it needs to be translated into a statistical model that allows for the testing and making of inferences about the hypothesized processes. Two approaches to achieving this goal have been developed in recent years. First, multilevel regression approaches allow for the simultaneous modeling of individual differences in average levels, variabilities, and autocorrelations of affective states that reflect the baselines, variabilities, and return to baseline of the DynAffect model, respectively (Wang, Hamaker, \& Bergeman, 2012). While over the years, several studies have employed multilevel regression models to analyze e-diary data in BPD, our approach is new in the sense that this study is the first to model several components of dysregulation in BPD simultaneously using these models. Second, Oravecz, Tuerlinckx, and Vandekerckhove (2011) developed a hierarchical (i.e., random effects) extension of a diffusion model based on stochastic differential equations that simultaneously captures the same set of parameters. While it is beyond the scope of this paper to explain these statistical models and their relationship in detail, it is important to emphasize that the models represent direct mathematical conceptualizations of the principles and assumptions of the DynAffect model (Kuppens, Oravecz, \& Tuerlinckx, 2010; see also below).

\section{Hypotheses}

For the empirical portion of this paper, we applied the proposed framework (i.e., the DynAffect model, ambulatory assessment, and the multilevel regression and diffusion modeling approaches) to three independent datasets. In all three studies, we used e-diaries to collect information about affective experiences elicited by natural stimuli in personally relevant environments from patients with BPD and healthy controls (HCs). The available data from these studies enabled the examination of affect in terms of valence and distress (Studies 1 and 3) or tense arousal (Study 2). While this deviates from the original DynAffect formulation in terms of valence and neutral arousal, the data nevertheless allow us to pinpoint the dynamic processes underlying fluctuations in multiple dimensions. To extract the subcomponents of the DynAffect model (i.e., affective homebases, variabilities, and attractor strengths), we used both multilevel regression and diffusion modeling. We made the following hypotheses for all three datasets: (a) the patients with BPD would report a more negative affective homebase than would the HCs, (b) compared to the HCs, the patients with BPD would exhibit heightened affective variability, and (c) compared to the HCs, the patients with BPD would exhibit reduced attractor strength (which would reflect a slower return to baseline). Since the three datasets are fairly similar in design and setup, we discuss their methods and findings simultaneously.

\section{Method}

\section{Participants: Dataset 1}

Fifty female patients with BPD and 50 female HCs were recruited from two sites: the University of Washington, Seattle, U.S.A. (42\%) and the University of Freiburg, Germany (58\%). Outpatients and inpatients were recruited from their outpatient clinics or wards. The Seattle HCs were recruited through advertisements, and the Freiburg HCs were selected randomly from the national resident register of the City of Freiburg. After the initial diagnostic procedure, all participants were carefully instructed and trained regarding the use of the e-diary (MONITOR software; Fahrenberg, Huettner, \& Leonhart, 2001) and carried a palmtop computer (Psion 3a, London, U.K.) for $24 \mathrm{hr}$. All subjects were paid for participation in the study and provided written informed consent. The study was approved by the respective ethical review committees of the University of Freiburg and the University of Washington. We refer the reader to several publications about the specific aspects of this dataset for further details (recall bias: Ebner-Priemer et al., 2006; physiological reactivity in daily life: Ebner-Priemer et al., 2007b; and affective instability: EbnerPriemer et al., 2007a). To perform the proposed analyses, the participants that lacked variability in their scores for one or both of the two key variables (i.e., valence and distress) were removed from the dataset. The resulting dataset consisted of 50 BPD patients and 48 HCs. The participants' sample characteristics are summarized in Table 1.

\section{Participants: Dataset 2}

Forty-two female patients with BPD and 24 female HCs were recruited from the Central Institute of Mental Health (CIMH), Mannheim, Germany. All patients were waiting for inpatient treatment. The HCs were selected randomly from the national resident register of the City of Mannheim or recruited via advertisement. After the initial diagnostic procedure, all participants were carefully instructed and trained regarding the use of the e-diary (Izy- 
Table 1

Demographic Characteristics for Borderline Personality Disorder (BPD)

\begin{tabular}{|c|c|c|c|c|c|c|}
\hline & \multicolumn{2}{|l|}{ Dataset 1} & \multicolumn{2}{|l|}{ Dataset 2} & \multicolumn{2}{|l|}{ Dataset 3} \\
\hline & $n=50$ BPD patients & $n=50 \mathrm{HC}$ & $n=42$ BPD patients & $n=24 \mathrm{HC}$ & $n=43$ BPD patients & $n=28 \mathrm{HC}$ \\
\hline \multicolumn{7}{|l|}{ Age in years } \\
\hline Mean $(S D)$ & $31.3(8.1)$ & $27.7(6.8)$ & $26.5(7.1)$ & $27.2(6.0)$ & $26.7(7.1)$ & $28.8(7.5)$ \\
\hline \multicolumn{7}{|l|}{ Sex } \\
\hline$\%$ Female & \multicolumn{2}{|c|}{ Dataset 1 ( $N=50$ BPD patients $)$} & \multicolumn{2}{|c|}{ Dataset 2 ( $N=42$ BPD patients) } & \multicolumn{2}{|c|}{$\begin{array}{c}100 \\
\text { Dataset } 3(N=43 \text { BPD patients })\end{array}$} \\
\hline $\begin{array}{l}\text { Psychotropic medication } \\
n(\%)\end{array}$ & \multicolumn{2}{|l|}{$40(80)$} & \multicolumn{2}{|l|}{$28(66.7)$} & \multicolumn{2}{|l|}{$16(37)$} \\
\hline \multicolumn{7}{|l|}{ Hospitalization } \\
\hline Outpatients $n(\%)$ & \multirow{2}{*}{\multicolumn{2}{|c|}{$\begin{array}{l}25(50) \\
25(50)\end{array}$}} & \multicolumn{2}{|l|}{$42(100)$} & \multicolumn{2}{|l|}{$26(60)$} \\
\hline Inpatients $n(\%)$ & & & $0(0)$ & & $17(40)$ & \\
\hline \multicolumn{7}{|l|}{ Current Axis I diagnoses $n(\%)$} \\
\hline Major depression & \multicolumn{2}{|l|}{$18(36)$} & \multicolumn{2}{|l|}{$26(61.9)^{\mathrm{a}}$} & \multicolumn{2}{|l|}{$9(21)$} \\
\hline Anxiety disorders & \multicolumn{2}{|l|}{$29(58)$} & \multicolumn{2}{|l|}{$23(54.8)$} & \multicolumn{2}{|l|}{$27(63)$} \\
\hline Generalized anxiety & \multicolumn{2}{|l|}{$1(2)$} & \multicolumn{2}{|l|}{$2(4.8)$} & \multicolumn{2}{|l|}{$6(14)$} \\
\hline Panic disorder & \multicolumn{2}{|l|}{$20(40)$} & \multicolumn{2}{|l|}{$12(28.6)$} & \multicolumn{2}{|l|}{$14(33)$} \\
\hline Agora phobia & \multicolumn{2}{|l|}{$1(2)$} & \multirow{2}{*}{\multicolumn{2}{|c|}{$3(7.1)$}} & \multirow{2}{*}{\multicolumn{2}{|c|}{$3(7)$}} \\
\hline Other phobias & \multirow{2}{*}{\multicolumn{2}{|c|}{$\begin{array}{l}20(40) \\
30(60)\end{array}$}} & $8(19)$ & & & $24(56)$ \\
\hline Posttraumatic stress disorder & & & $11(26.2)$ & & $22(51)$ & \\
\hline Obsessive-compulsive disorder & $1(2)$ & & $3(7.1)$ & & $5(11)$ & \\
\hline Eating disorders & $25(50)^{\mathrm{b}}$ & & $14(33.3)$ & & $14(33)$ & \\
\hline Current Axis II disorders $n(\%)$ & & & & & & \\
\hline Cluster A & $14(28)$ & & $9(21.4)$ & & $7(16.3)$ & \\
\hline Cluster B (besides BPD diagnosis) & $1(2)$ & & $1(2.4)$ & & $3(7.0)$ & \\
\hline Cluster C & $18(36)$ & & $25(59.5)$ & & $26(60.5)$ & \\
\hline
\end{tabular}

${ }^{\mathrm{a}}$ Major depression and recurrent depressive disorder. ${ }^{\mathrm{b}}$ Lifetime.

Builder software, IzyData Ltd., Fribourg, Switzerland) and carried a palmtop computer (Tungsten E, Palm Inc., U.S.A.) for 4 days. All subjects were paid for their participation in the study and provided written informed consent. The ethical review committee of the University of Heidelberg, Germany approved the study. Data from one healthy control participant had to be removed due to a lack of variability in her scores. Thus, the final sample consisted of 42 BPD patients and 23 HCs. The participants' sample characteristics are summarized in Table 1.

\section{Participants: Dataset 3}

Forty-three female patients with BPD and 28 female HCs were recruited from two sites in Germany: the CIMH and the Psychosomatic Clinic St. Franziska-Stift Bad Kreuznach. Outpatients and inpatients were recruited from their outpatient clinics or wards or via advertisements that were placed in local newspapers and on the Internet. The HCs were selected randomly from the national resident register of the City of Mannheim or via advertisement. After the initial diagnostic procedure, the patients were carefully instructed and trained regarding the use of the e-diary and carried a palmtop computer (Tungsten E, Palm Inc., U.S.A.) for $24 \mathrm{hr}$. Due to a standard rule of the two assessment sites, the patients who were admitted to treatment were not financially compensated. However, the patients who were not in treatment and HCs received financial compensation. The local ethics committee approved the study, and all participants provided written informed consent prior to participation. For further details about the dataset, we refer the reader to a publication about the specific aspects of this dataset (affective instability: Santangelo et al., 2014). Data from seven HCs were excluded from the analyses due to lack of variability in their ratings. The final sample consisted of 43 BPD patients and 21 HCs. The participants' sample characteristics are summarized in Table 1.

\section{Diagnostic Procedure: All Datasets}

Trained psychologists (Freiburg, Mannheim) and Master's-level clinical assessors (Seattle) administered the Structured Clinical Interview for DSM-IV Axis I Disorders (SCID-I: English version: First, Spitzer, Gibbon, Williams, \& Benjamin, 1997; German version: Wittchen, Wunderlich, \& Gruschwitz, 1997) and the International Personality Disorder Examination (IPDE: Loranger, 1999). We included patients aged between 18 and 45 years who met the DSM-IV diagnostic criteria for BPD. Patients with histories of schizophrenia, bipolar disorder, or current substance abuse were excluded. The exclusion criteria for the HC group included diagnoses of BPD (as assessed by the IPDE), any current or past Axis I disorder (SCID-I), current psychotherapy or current use of medication, and other Axis II disorders (SCID-II: Fydrich, Renneberg, Schmitz, \& Wittchen, 1997). The latter exclusion criterion applied only to the German sample.

\section{Diary Items and Time-Based Design: Datasets 1 and 3}

The e-diary software (Dataset 1: MONITOR: Fahrenberg et al., 2001; Dataset 3: DialogPad, Gerhard Mutz, Cologne University, Germany) emitted a prompting signal every $15 \mathrm{~min}$ ( $\pm 5 \mathrm{~min}$ ) only during the waking hours of a 24-hr period. Each response was automatically time-stamped. The two dependent variables of interest used in the statistical analyses were valence and distress. To obtain the valence scores, the participants rated the intensities of 
their primary and secondary emotions on an 11-point Likert scale. These rating were aggregated into a single valence index by multiplying the intensities of negative emotions (i.e., anxious, angry, shame, disgust, sad, guilt, envy/jealousy, and unpleasant unnamable emotion) by -1 and multiplying the intensities of the positive emotions (i.e., happy, interest, and pleasant unnamable emotion) by +1 (for details, see Ebner-Priemer et al., 2007a). Additionally, the participants rated the current intensity of their distress on a single 11-point Likert scale that ranged from 0 to 10.

\section{E-Diary Items And Time-Based Design: Dataset 2}

The e-diary software (IzyBuilder, IzyData Ltd., Fribourg, Switzerland) emitted a prompting signal every $60 \mathrm{~min}( \pm 10 \mathrm{~min})$ during the waking hours of a 4-day period. Each response was automatically time-stamped. A six-item short scale of the Multidimensional Mood Questionnaire that was explicitly developed and evaluated for ambulatory assessment was used (Wilhelm \& Schoebi, 2007). In the analyses, we used the two subscales of valence (unwell vs. well) and tense arousal (relaxed vs. tense) and two bipolar items for each subscale. The tense arousal scale serves as a proxy for distress. The subscale scores range from 0 (low value) to 6 (high value).

\section{Statistical Analyses}

According to the DynAffect model, a person's changes in core affect are driven by three components: (a) the level of the affective homebase (which reflects one's affective baseline state), (b) affective variability (which reflects the total sum of the changes around the affective homebase in response to internal or external events), and (c) attractor strength (which reflects the regulatory processes that pull core affect back to its homebase). For all three datasets, these processes were modeled with both multilevel regression modeling and diffusion modeling approaches to enable the identification of consistent patterns of results across the different modeling approaches and datasets and to allow for robust conclusions (i.e., conclusions that were not functions of specific modeling choices or datasets).

Multilevel regression models were separately estimated for valence and distress to simultaneously model the three different parameters proposed by the DynAffect model and to investigate how these parameters differed as functions of BPD diagnosis. Analyses were performed using HLM (Raudenbush, Bryk, \& Congdon, 2004), and these analyses were performed for all three datasets (thus, a total of six models were estimated). The models were defined as follows:

Level 1 equation:

$$
Y_{\mathrm{ti}}=\pi_{0 \mathrm{i}}+\pi_{1 \mathrm{i}} * Y_{(\mathrm{t}-1) \mathrm{i}}+e_{\mathrm{ti}}
$$

Level 2 equation:

$$
\begin{aligned}
\pi_{0 \mathrm{i}} & =\beta_{00}+\beta_{01} *\left(X_{\mathrm{i}}\right)+r_{0 \mathrm{i}} \\
\pi_{1 \mathrm{i}} & =\beta_{10}+\beta_{11} *\left(X_{\mathrm{i}}\right)+r_{1 \mathrm{i}} \\
\operatorname{Var}(\mathrm{R}) & =\sigma^{2} \text { and } \log \left(\sigma^{2}\right)=\alpha_{0}+\alpha_{1}\left(X_{i}\right)
\end{aligned}
$$

where $Y_{\mathrm{ti}}$ is the assessment of one of the dimensions (valence or distress) for person $i$ at time $t$. At Level $1, Y_{\mathrm{ti}}$ is predicted by a random intercept, and a time-lagged version of itself $Y_{(\mathrm{t}-1) \mathrm{i}}$ (which was within-person centered; previous-day observations were set as missing to avoid the inclusion of day-to-day carry-over effects). The random slope of this lagged variable, $\pi_{1 \mathrm{i}}$, is the autoregressive effect of $Y_{(\mathrm{t}-1) \mathrm{i}}$ on $Y_{\mathrm{ti}}$. At Level 2, the random intercept and slope are both predicted by an intercept and the BPD dummy variable $X_{\mathrm{i}}$, which codes for BPD $\left(X_{\mathrm{i}}=1\right)$ or $\operatorname{HCs}\left(X_{\mathrm{i}}=0\right)$. Simultaneously, the within-person variance is modeled as a function of the BPD dummy.

Such a model incorporates the three basic processes involved in the DynAffect model in the following manner. First, the intercept at Level $1, \pi_{0 i}$, represents the mean value for $Y$ for each individual and therefore reflects the affective homebase for that dimension. At Level 2, this intercept, $\pi_{\mathrm{Oi}}$, is modeled as a function of BPD, with $\beta_{01}$ indicating whether the affective homebase significantly differs between the BPD and the $\mathrm{HC}$ group (note that $\beta_{00}$ is an estimate for the mean level of $Y$ for the HCs (when $X_{\mathrm{i}}=0$ ) and that the sum of $\beta_{00}$ and $\beta_{01}$ reflects the mean level of $Y$ for the patients with BPD (when $\left.X_{\mathrm{i}}=1\right)$ ).

Second, within-person variance reflects the affective variability component. This variance is modeled as a function of the BPD dummy variable, with $\alpha_{1}$ providing an estimate of the group difference between the Level 1 variance of the HCs and patients with BPD (note that $\alpha_{0}$ is the Level 1 variance for the HCs (when $X_{\mathrm{i}}=0$ ) and that the sum of $\alpha_{0}$ and $\alpha_{1}$ reflects the Level 1 variance of the patients with BPD (when $\left.X_{\mathrm{i}}=1\right)$ ).

Third, $\pi_{1 i}$ is the autoregressive slope at Level 1 and inversely reflects the attractor strength (Oravecz et al., 2011). Again, at Level 2, the autoregressive slope, $\pi_{1 \mathrm{i}}$, is modeled as a function of $X_{\mathrm{i}}$, the BPD dummy, with $\beta_{11}$ providing an estimate of the group difference between the HCs and the patients with BPD in terms of attractor strength (note that $\beta_{10}$ reflects the autocorrelation for the HCs when $X_{\mathrm{i}}=0$ and that the sum of $\beta_{10}$ and $\beta_{11}$ is the autocorrelation for the patients with BPD when $X_{\mathrm{i}}=1$ ).

In addition to the multilevel regression analyses, a hierarchical latent diffusion model (Kuppens, Oravecz, \& Tuerlinckx, 2010; Oravecz et al., 2011) was also used to model core affect fluctuations over time in all three data sets. The latent diffusion model is a continuous-time statistical model for measurements taken at possibly irregular time points (as is the case for the current data). This is in contrast to the multilevel models which assume equal time intervals between time points. Similarly, as in the multilevel regression models, the person-specific parameters are assumed to be sampled from a population distribution (i.e., they are random effects), which accounts for individual differences. The personspecific parameters of the diffusion model are the homebase, the variability, and attractor strength. However, unlike the regression analyses, this statistical diffusion model estimates the three parameters of both dimensions (instead of one dimension) simultaneously, which allows for covariation between the two dimensions to be considered. In addition, it is possible to include person covariates into the diffusion model, such as BPD diagnostic status. Using covariates, it becomes possible to explain individual differences in parameters by regressing them on covariates (e.g., regarding BPD diagnostic status, the person-specific parameters can differ systematically between the BPD patients and the healthy participants). Moreover, the traditional multilevel regression analyses are based on a frequentist statistical approach, while the diffusion model uses Bayesian inference. For detailed statistical information about the 
diffusion model and statistical software to run the analyses, see Oravecz et al. (2011), and Oravecz, Tuerlinckx, \& Vandekerckhove (2012)

These differences in approaches may give rise to slight differences in results, allowing us to identify findings that are robust across methods and distinguish them from findings that may be specific to one particular approach and, therefore, possibly less reliable.

Moreover, to graphically depict the data, vector fields were separately estimated for each group in each dataset. After pooling the data across the entire group, the core affect space was divided into cells in which each cell represented some combination of valence and distress, ${ }^{1}$ and a vector was calculated for each cell. The length of the vector reflects the average speed of escape from the cell (i.e., if a person is in a certain emotional state, how fast is that likely to change), while the direction reflects, on average, how a person will feel next (Kuppens, Oravecz, \& Tuerlinckx, 2010). When the duration between consecutive time points was five hours or more, the corresponding data points were not used to compute vectors.

\section{Results}

In this section, we will simultaneously report the results for all three datasets ${ }^{2}$ from the multilevel regression models as the primary results. Subsequently, these results will briefly be compared to those from the diffusion modeling approach to identify consistencies across both approaches.

\section{Affective Homebase}

In all three datasets, the results for the valence dimension of the homebase revealed significant differences between patients with BPD and $\mathrm{HCs}\left(X_{\mathrm{i}}, \beta_{01}\right.$, Table 2$)$. While the HCs were, on average, characterized by a pleasant or positive homebase, the patients with BPD tended to have an unpleasant or negative homebase. These results are graphically depicted in Figure 1. In each vector field, the large red dot marks the affective homebase. The affective homebase of the BPD patients is located within the negative affective range of the vector fields (see Figure 1a), whereas the affective homebase of the HCs is located within the positive affective range (Figure 1b).

The results regarding distress from all three datasets revealed that the patients with BPD were characterized by homebases that involved significantly higher levels of distress $\left(X_{\mathrm{i}}, \beta_{01}\right)$ compared to those of HCs (see Table 3). These results are illustrated in Figure 1, showing the homebase of the BPD patients being located farther up in the vector fields (Figure 1a) than the homebase of the HCs (Figure 1b).

\section{Affective Variability}

In terms of variability in valence and distress, the two groups differed significantly in all three datasets. The patients with BPD exhibited greater variability in the valence dimension $\left(X_{\mathrm{i}}, \alpha_{1}\right.$; Table 2), and in the distress dimension $\left(X_{\mathrm{i}}, \alpha_{1}\right.$; Table 3$)$ than the $\mathrm{HCs}$ across all datasets. Figure 1 graphically depicts these elevated affective variabilities of the BPD patients via the larger dispersion of affective states around the homebase of the BPD patients (Figure 1a) than around the homebase of the HCs (Figure 1b).

\section{Attractor Strength (Return to Baseline)}

The results regarding attractor strength were less consistent across datasets. For valence, we found a significant difference between the BPD patients and the healthy participants in the autoregressive slope (i.e., the reverse of attractor strength), but only in dataset 2 . These findings demonstrated that BPD patients were characterized by a higher autoregressive slope for valence $\left(X_{\mathrm{i}}, \beta_{11}\right)$ than were the HCs, which reflects lower attractor strength and a slower return to baseline for valence among BPD patients. However, no significant differences in autoregressive slope for valence were found between the patients with BPD and HCs in Datasets 1 and 3 (see Table 2).

Regarding distress, Datasets 1 and 3 revealed higher autoregressive slopes for BPD patients than for the healthy participants $\left(X_{\mathrm{i}}\right.$, $\beta_{11}$ ), indicating that the BPD patients were characterized by a lower attractor strength or slower return to baseline for distress than the HCs. No difference in autoregressive slope and thus attractor strength for distress was found in Dataset 2 (see Table 3$)^{3}$.

The return to baseline or attractor strengths are graphically depicted in Figure 1 in the form of vector fields. After pooling the data from participants from each subgroup separately, we calculated a vector for each cell that represents an affective state. The length of the vector is proportional to the average speed of escape away from that affective state, and the direction of the vector indicates the direction of the escape. As can be seen from the plots, the farther a participant's current feeling state is away from the affective homebase, the higher the escape speed toward the homebase becomes, as shown by longer vectors for states that deviate more from the homebase. Additionally, most of the vectors point nearly directly toward the affective homebase.

Visual inspection of the vector plots revealed that, for BPD patients, the lengths of the vectors (which reflect return to baseline) seemed to be larger for the emotion states that are more positive than the average homebase compared to those for the emotion states that are more negative than the homebase, while healthy individuals rather show the opposite pattern. Thus, the vector plots seemed to indicate that the BPD individuals exhibited stronger return to baseline from positive than from negative states and that the healthy indi-

\footnotetext{
${ }^{1}$ Please note that unlike the two dimensions of core affect-valence and arousal-valence and distress as used in our analyses are not orthogonal, as might be suggested by the square plots. However, square plots were merely used for ease of visualization and interpretation.

${ }^{2}$ Dataset 1 involves participants studied at two different locations: Germany and U.S.A. To test whether location had any meaningful impact on the results, we estimated all models on the samples of each country separately, obtaining largely similar results.

${ }^{3}$ It should be noted that an assumption of the reported multilevel regression analyses that were performed using HLM (Raudenbush et al., 2004 ) is that the within-person variance is homogenous within groups. To evaluate whether results remain the same when assuming heterogeneous within-person variance, we performed additional Bayesian multilevel analyses using Winbugs based on the model described by Wang et al. (2012), in which, next to the intercept and autoregressive slope, also within-person variance was estimated as a random effect. Results from the two different types of multilevel analyses were consistent with two small exceptions: the Bayesian multilevel analyses indicated that the within-person variance of HCs for distress in Dataset 1 and the distress level of the home base of the HCs in Dataset 3 were unlikely to differ from 0. Most importantly, however, the results were consistent concerning the differences between the BPD and HCs in home base, variability, and attractor strength.
} 
Table 2

Estimates From Multilevel Models for all Datasets for the Valence Dimension

\begin{tabular}{|c|c|c|c|c|c|c|c|c|c|c|c|c|c|c|c|}
\hline \multirow[b]{2}{*}{ Valence } & \multicolumn{5}{|c|}{ Dataset 1} & \multicolumn{5}{|c|}{ Dataset 2} & \multicolumn{5}{|c|}{ Dataset 3} \\
\hline & Coeff. & $S E$ & $\begin{array}{l}\text { Test } \\
\text { statistic }\end{array}$ & $d f$ & $p$-value & Coeff. & $S E$ & $\begin{array}{l}\text { Test } \\
\text { statistic }\end{array}$ & $d f$ & $p$-value & Coeff. & $S E$ & $\begin{array}{l}\text { Test } \\
\text { statistic }\end{array}$ & $d f$ & $p$-value \\
\hline \multicolumn{16}{|l|}{ For homebase, $\pi_{0}$} \\
\hline Intercept, $\beta_{00}$ & 1.46 & 0.15 & 9.96 & 96 & $<.001$ & 5.02 & 0.13 & 40.07 & 63 & $<.001$ & 1.67 & 0.29 & 5.68 & 62 & $<.001$ \\
\hline$X_{i}, \beta_{01}$ & -2.56 & 0.27 & -9.47 & 96 & $<.001$ & -2.24 & 0.20 & -11.05 & 63 & $<.001$ & -4.25 & 0.50 & -8.43 & 62 & $<.001$ \\
\hline \multicolumn{16}{|l|}{ For variance } \\
\hline Intercept, $\alpha_{0}$ & 1.94 & 0.03 & 67.29 & & $<.001$ & -0.38 & 0.05 & -7.77 & & $<.001$ & 2.08 & 0.04 & 46.87 & & $<.001$ \\
\hline$X_{i}, \alpha_{1}$ & 0.43 & 0.04 & 10.48 & & $<.001$ & 0.87 & 0.06 & 14.27 & & $<.001$ & 1.06 & 0.05 & 19.52 & & $<.001$ \\
\hline $\begin{array}{l}\text { For autoregressive slope, } \pi_{1} \\
\text { Intercept, } \beta_{10}\end{array}$ & 0.24 & 0.03 & 8.57 & 96 & $<.001$ & 0.23 & 0.04 & 5.08 & 63 & $<.001$ & 0.26 & 0.05 & 5.75 & 62 & $<.001$ \\
\hline$X_{i}, \beta_{11}$ & 0.07 & 0.04 & 1.53 & 96 & .129 & 0.18 & 0.06 & 3.13 & 63 & .003 & 0.06 & 0.06 & 1.08 & 62 & .283 \\
\hline
\end{tabular}

Note. $\quad$ Coeff. $=$ coefficient. The reported degrees of freedom are approximations. Coefficients concerning the level 1 intercept and autoregressive slope are tested using a $t$-ratio. Coefficients concerning the variance are tested using a $Z$-ratio.

viduals exhibited stronger return to baseline from negative than from positive states. To statistically test this notion, we performed a two-way analysis of variance on the lengths of all vectors (which were logtransformed to approximate a normal distribution) to test whether the average vector lengths differed between states that were more positive and more negative than the homebase (i.e., their locations in the affect grid) and whether this effect differed between the BPD patients and the HCs.

While no significant location* group interaction on vector length was observed in Dataset 1 (see below), we found significant and marginally significant interactions in Datasets $3, F(1,281)=5.41$, $p=.021$ and $2, F(1,209)=3.40, p=.067$, respectively, between group and location in the affect grid on the length of the vectors. Further examination of this difference revealed that the vector lengths of the BPD patients were larger when they were in emotional states that were more positive than their affective baselines or homebases $(M=0.20, S E=0.01$ in Dataset 2; $M=0.50, S E=$ 0.03 in Dataset 3) than when they were in states more negative than their homebases $(M=0.17, S E=0.02$ in Dataset $2 ; M=$ $0.36, S E=0.03$ in Dataset 3). This difference was significant in Dataset $3(p \leq .001)$ but not in Dataset $2(p=.121)$. For the HCs, no significant effects of location in the affect grid were found in Datasets $2(p=.262)$ or $3(p=.581)$. In Dataset 1 , we observed only a main effect of group, $F(1,300)=3.86, p=.050$. The HCs $(M=0.50, S E=0.03)$ returned to baseline more quickly than did the BPD patients $(M=0.43, S E=0.02)$, and this finding is consistent with our main analyses.

These results suggest that, in BPD, the attractor strength or the return to baseline can depend on the valence of the emotional state that a person is in. More specifically, if persons with BPD are in states that are more positive than how they feel on average, they tend to return to baseline more quickly than when they are in more negative states. However, since results were mixed across datasets, they should be interpreted with caution. Future studies investigating this phenomenon in more detail should be conducted.

\section{Diffusion Modeling Results}

In addition to the multilevel regression analyses reported above, we performed a hierarchical diffusion model similar to the one described in Kuppens, Oravecz, and Tuerlinckx (2010) using a
Bayesian approach and custom-made software (Oravecz et al., 2011; Oravecz et al., 2012). The results produced by this model led to conclusions regarding the homebases and variabilities of the two dimensions that were consistent with the results reported above. Regarding attractor strength, the hierarchical diffusion model produced somewhat different results; no differences related to the attractor strengths of any of the dimensions were found in Datasets 1 and 3. The results from Dataset 2 were consistent with the multilevel regression results, but these results were based on a model that did not reach satisfactory convergence. This discrepancy in results is most likely due to one or more of the differences in statistical approaches as explained in the method section, and indicate that results for attractor strength not only differ as a function of dimension and dataset, but also as a function of analysis method.

\section{Discussion}

Our paper addressed two topics. First, we argue that to better understand affective dysregulation, an overarching theoretical model of emotional dynamics that involves multiple components of dysregulation, an assessment method to gather data in response to multiple personally relevant stimuli, and an adequate statistical model are needed. Second, based on these arguments, we use a multilevel modeling approach that is based on the DynAffect model to investigate three components of affective dysregulation simultaneously using three separate BPD/HC datasets. To our knowledge, there is, at present, no published paper that proposes such a framework and uses multilevel modeling to simultaneously analyze several components (homebase, variability, return to baseline) of affective dysregulation.

The results from all three datasets regarding affective homebase were consistent. Compared to the HCs, the patients with BPD were characterized by a homebase that was more negative and involved a greater level of distress. Thus, our findings are in agreement with the suggestion of Linehan's (1993) biosocial theory that BPD is accompanied by heightened sensitivity to particularly negative emotional stimuli. Heightened sensitivity to negative emotional stimuli should lead to more negative and distressed affective states, and these states characterize the affective homebase of a subject. Comparison of our findings to those of previous studies is difficult 
$A(B P D)$

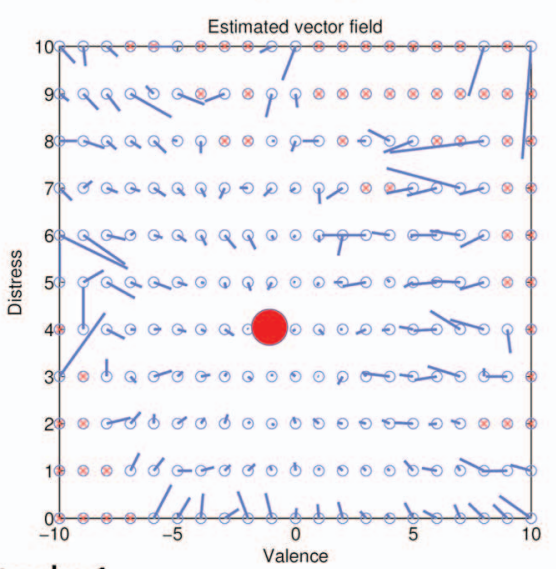

Study 1
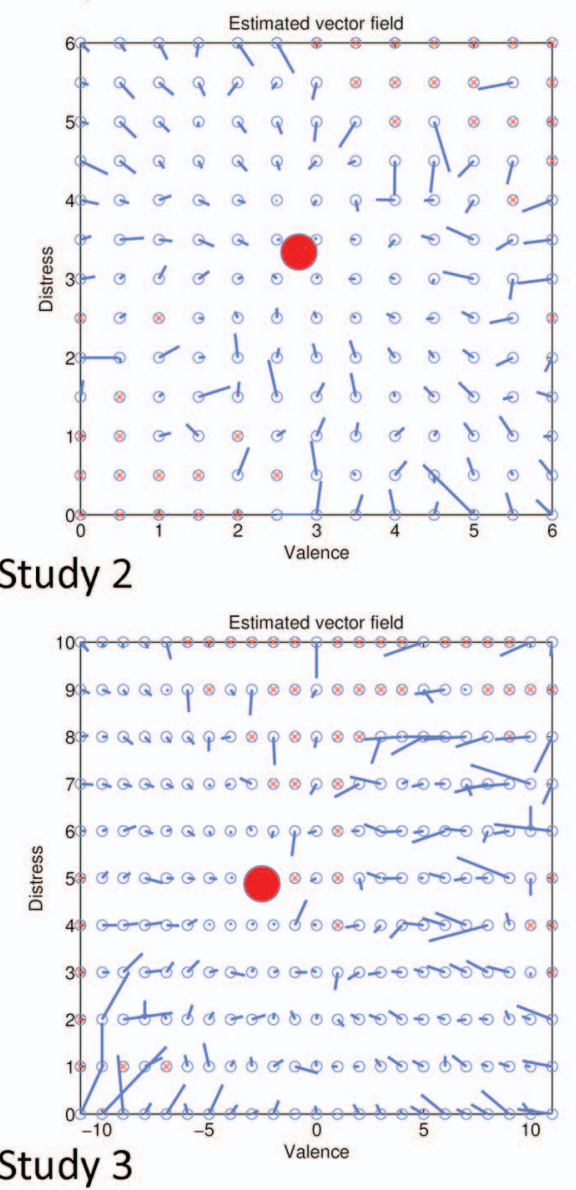

$\mathrm{B}$ (HCS)
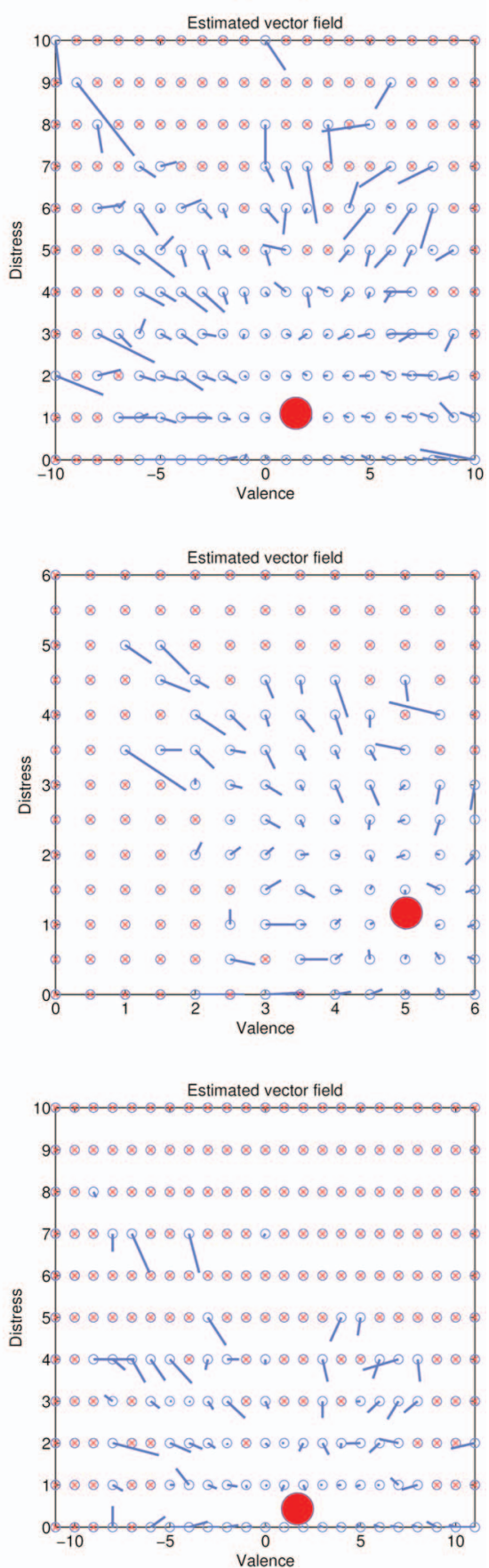

Figure 1. Vector fields depicting aggregated data from BPD patients (a) and healthy controls (b) in Studies 1, 2 , and 3. The red dot indicates the location of the home base. The length of the vector for each combination of valence and distress is proportional to the average speed of escape from that position. The direction of the vector reflects the direction of the escape. Missing data are indicated by a cross.

because we calculated several affective components simultaneously (i.e., homebase, variability, and return to baseline) in one statistical model, whereas other studies have calculated each effect (i.e., baseline, reactivity, etc.) in stand-alone analyses. However, heightened negative and distressed affective baseline values in patients with BPD have been reported in studies that have utilized film clips (Elices et al., 2012; Kuo \& Linehan, 2009; Staebler et al., 2009) and short stories (Jacob et al., 2009) and in laboratory 
Table 3

Estimates From Multilevel Models for all Datasets for the Distress Dimension

\begin{tabular}{|c|c|c|c|c|c|c|c|c|c|c|c|c|c|c|c|}
\hline \multirow[b]{2}{*}{ Distress } & \multicolumn{5}{|c|}{ Dataset 1} & \multicolumn{5}{|c|}{ Dataset 2} & \multicolumn{5}{|c|}{ Dataset 3} \\
\hline & Coeff. & $S E$ & $\begin{array}{c}\text { Test } \\
\text { statistic }\end{array}$ & $d f$ & $p$-value & Coeff. & $S E$ & $\begin{array}{c}\text { Test } \\
\text { statistic }\end{array}$ & $d f$ & $p$-value & Coeff. & $S E$ & $\begin{array}{c}\text { Test } \\
\text { statistic }\end{array}$ & $d f$ & $p$-value \\
\hline \multicolumn{16}{|l|}{ For homebase, $\pi_{0}$} \\
\hline Intercept, $\beta_{00}$ & 1.11 & 0.15 & 7.44 & 96 & $<.001$ & 1.16 & 0.15 & 7.62 & 63 & $<.001$ & 0.44 & 0.11 & 4.05 & 62 & $<.001$ \\
\hline$X_{i}, \beta_{01}$ & 2.94 & 0.27 & 10.79 & 96 & $<.001$ & 2.17 & 0.21 & 10.24 & 63 & $<.001$ & 4.44 & 0.30 & 14.84 & 62 & $<.001$ \\
\hline \multicolumn{16}{|l|}{ For variance } \\
\hline Intercept, $\alpha_{0}$ & 0.20 & 0.03 & 7.00 & & $<.001$ & -0.27 & 0.05 & -5.57 & & $<.001$ & -0.28 & 0.04 & -6.37 & & $<.001$ \\
\hline$X_{i}, \alpha_{1}$ & 0.90 & 0.04 & 21.71 & & $<.001$ & 0.71 & 0.06 & 11.65 & & $<.001$ & 1.49 & 0.05 & 27.36 & & $<.001$ \\
\hline \multirow{2}{*}{$\begin{array}{l}\text { For autoregressive slope, } \pi_{1} \\
\text { Intercept, } \beta_{10} \\
X_{i}, \beta_{11}\end{array}$} & & & & & & & & & & & & & & & \\
\hline & $\begin{array}{l}0.29 \\
0.12\end{array}$ & $\begin{array}{l}0.03 \\
0.05\end{array}$ & $\begin{array}{l}8.99 \\
2.53\end{array}$ & $\begin{array}{l}96 \\
96\end{array}$ & $\begin{array}{r}<.001 \\
.013\end{array}$ & $\begin{array}{l}0.30 \\
0.07\end{array}$ & $\begin{array}{l}0.04 \\
0.06\end{array}$ & $\begin{array}{l}6.98 \\
1.20\end{array}$ & 63 & $\begin{array}{r}<.001 \\
234\end{array}$ & $\begin{array}{l}0.29 \\
0.18\end{array}$ & 0.06 & $\begin{array}{l}5.02 \\
250\end{array}$ & 62 & $<.001$ \\
\hline
\end{tabular}

Note. $\quad$ Coeff. $=$ coefficient. The reported degrees of freedom are approximations. Coefficients concerning the level 1 intercept and autoregressive slope are tested using a $t$-ratio. Coefficients concerning the variance are tested using a Z-ratio.

studies that have used interpersonal stressors (Reitz et al., 2012). Another important difference between our approach and those of previous laboratory studies is the definition of baseline. In laboratory studies, the baseline is most frequently defined by the absence of standardized stimuli. The participants wait, possibly while connected to wires, until the experiment begins. Whether the participants are bored, exhibiting anticipatory anxiety, or ruminating is unclear. In contrast, in our studies, the affective homebase was defined by the normative affective state of the participant as the participant was faced with the normal affective stimuli of daily life. Thus, we suggest that this definition of the affective homebase has higher ecological validity and clinical relevance than does the definition of the homebase as a nonstimuli baseline. Again, for correctness of interpretation, a more negative or distressed affective homebase does not imply that patients with BPD intentionally prefer more negative feelings. It does, however, imply that the state to which their feelings are attracted to is of a more negative valence.

Regarding affective variability, the patients with BPD exhibited consistently higher variability in both valence and distress than the $\mathrm{HCs}$ in all three datasets. Again, these findings are in agreement with the notion of intense responses to emotional stimuli in BPD that was proposed by Linehan (1993). Intense responses to emotional stimuli should result in a heightened variability. Previous studies have produced mixed findings regarding heightened affective reactivity; whereas some studies have revealed heightened reactivity (Donegan et al., 2003; Ebner-Priemer et al., 2005; Herpertz et al., 2001; Koenigsberg et al., 2009), others have reported hyporeactivity in BPD (Herpertz et al., 1999). Again, when our findings are compared to those of previous studies, the fact that we simultaneously calculated all three affective components (homebase, variability, and return to baseline) in one statistical model, while the other studies calculated each effect separately, must be remembered. Additionally, our studies collected data in personally relevant environments and thus our results have greater ecological validity.

The results regarding attractor strength were less consistent. In Sample 2, we found that the patients with BPD exhibited lower attractor strengths for valence than the HCs. The results from Samples 1 and 3 indicated that the patients with BPD exhibited lower attractor strengths for distress (additionally, the results from the alternative diffusion model analyses were inconsistent with those from the main analyses due to the mentioned differences in statistical approach). Moreover, it should be acknowledged that, if there were any differences in attractor strength between the BPD and healthy individuals, they were clearly of smaller magnitudes than those observed for homebase and affective variability. This points to the conclusion that differences between BPD patients and $\mathrm{HCs}$ in terms of attractor strength are less reliable and of smaller magnitude compared to differences in homebase and affective variability. That being said, although the samples did not exhibit significant differences in attractor strength for each of the dimensions, all of the estimations pointed to lower attractor strengths in BPD. Therefore, we conclude that our data provide only weak support for the slow return to baseline proposed in Linehan's biosocial theory (Linehan, 1993). This slow return to baseline has been investigated only rarely in previous studies and has been shown to be particularly prominent for specific emotions such as shame (Gratz et al., 2010), anxiety, and sadness (Reisch et al., 2008). Based on the current data, which were collected in people's daily lives, we can conclude that BPD patients exhibit signs of slower return to baseline, but this is not the most characteristic feature of their emotion dynamics. Additionally, we found some evidence that the return to baseline in BPD patients does depend on the current affective state; the return to baseline seemed stronger when the patients were in positive states than when they were in negative states. This finding is in accordance with the clinical picture of BPD patients, which assumes that BPD patients can transition abruptly from positive mood states to very negative mood states and can remain in negative mood states for extended periods. However, more research is needed to examine this pattern in more detail.

In the introduction, we proposed that affective processes in psychiatric disorders, such as affective dysregulation in BPD, should be investigated in the context of an overarching theoretical model with a method that assesses affective processes in a personally relevant environment and with appropriate statistical methods. We tied this claim to the hope that such investigations would enable the use of the progress in basic affective science and enable comparison of mechanisms and processes across studies and across disorders. Thus, the transdiagnostic nature of our approach may help to elucidate the common and distinctive mechanisms that 
underlie several different disorders that are characterized by affective dysregulation.

Despite the numerous strengths of this study that include the combination of basic affective science theory, ecological data, and appropriate statistical modeling and the importance of replication across datasets (Begley \& Ellis, 2012), some limitations should be mentioned. First, we only assessed female patients and thus the generalizability of our findings is limited. However, given the literature on sex differences on emotion (Fischer, 2000), a pure female sample reduces heterogeneity, which may be useful.

Second, the operationalizations of valence and distress varied slightly across the three datasets. Whereas valence was assessed with a categorical approach in Datasets 1 and 3, we used a dimensional approach for dataset 2. Additionally, the time-based designs differed across the datasets; assessments occurred every 15 min in Datasets 1 and 3, whereas queries were made every hour in Dataset 2. Nevertheless, the conclusions were largely similar across datasets, which suggests that the assessment methods did not substantially influence the results. Moreover, we are confident that both sample designs were optimal to assess current levels of homebase, variability, and attractor strength, since these designs led to 40 to 70 repeated assessments per person over the course of a day (or days), with a high frequency sampling rate with 15 or 60 min intervals between measurements. As shown by Ebner-Priemer and Sawitzki (2007), which addressed the influence of different time intervals on the affective instability of patients with BPD, high-frequency sampling rates (15 min and $30 \mathrm{~min}$ ) track a specific process, whereas the data yielded by low frequency sampling rates ( $2 \mathrm{hr}$ and $4 \mathrm{hr}$ ) cannot be distinguished from random data. Given this information and the general notion that, when in doubt, it is better to use shorter intervals (Bolger, Davis, \& Rafaeli, 2003), we assume that our sampling frequency was appropriate. However, in the future, we need more studies to investigate how time-based designs exactly influence the outcomes in diary studies. In addition, we assessed distress in Dataset 1 and Dataset 3, while we assessed tense arousal in Dataset 2. The use of distress instead of arousal questions the assumption of orthogonality, as proposed in the original Russell (2003) core affect model. However, whether dependence between valence and distress/tense arousal was (in the statistical diffusion model) or was not (in the multilevel regression model) taken into account did not largely affect the results, since results from both analyses are in line with the conclusion we draw.

Third, we should note that the estimates of homebase, variability, and attractor strength refer to current state levels of these parameters that are characteristic of a person. This does not imply that these parameters should be considered stable traits that cannot change over time. For example, they could change as a function of the developmental stage of a disorder. Yet, for the current purpose, the aim is to provide an estimate of people's current levels. Similarly, it might be the case that BPD patients are characterized by two instead of one affective homebase and, consequently, switch between different homebase states over time. However, at this point, the statistical tools are still lacking for modeling such complex dynamic systems, and more research is needed to address these empirical questions in the future.

Fourth, because we did not use clinical control groups in our analyses, the specificities of our findings remain unclear. It is also unclear whether our group differences are related to BPD or can be explained by comorbid disorders. Clearly, studies with clinical control groups are needed (see Santangelo et al., 2014 for a detailed discussion).

As highlighted in our paper, sophisticated behavioral science research methods for the investigation of psychological mechanisms are available for the study of psychopathology in daily life. While current initiatives to fundamentally improve psychopathological research are looking more at basic processes spanning across disorders, such as the Research Domain Criteria of the National Institutes of Health (Cuthbert \& Insel, 2013; Cuthbert \& Kozak, 2013), they do fall short in understanding human behaviors as dynamical processes that unfold in the broadest setting imaginable-everyday life. Only the combination of overarching theoretical models, findings from basic affective science, and sophisticated statistical models and methods that are capable of assessing dynamical affective mechanisms in everyday life will help to close this gap. Such combinations will enable us to consider psychopathology in terms of dysregulations and dysfunctions of the fundamental aspects of behavior and affect, to investigate and compare the fundamental psychological dimensions that cut across traditional disorders, and, in the long run, help to develop more specific treatment modules.

\section{References}

Austin, M. A., Riniolo, T. C., \& Porges, S. W. (2007). Borderline personality disorder and emotion regulation: Insights from the Polyvagal Theory. Brain and Cognition, 65, 69-76. http://dx.doi.org/10.1016/j.bandc 2006.05.007

Begley, C. G., \& Ellis, L. M. (2012). Drug development: Raise standards for preclinical cancer research. Nature, 483, 531-533. http://dx.doi.org/ $10.1038 / 483531$ a

Bolger, N., Davis, A., \& Rafaeli, E. (2003). Diary methods: Capturing life as it is lived. Annual Review of Psychology, 54, 579-616. http://dx.doi .org/10.1146/annurev.psych.54.101601.145030

Carpenter, R. W., \& Trull, T. J. (2013). Components of emotion dysregulation in borderline personality disorder: A review. Current Psychiatry Reports, 15, 335. http://dx.doi.org/10.1007/s11920-012-0335-2

Crowell, S. E., Beauchaine, T. P., McCauley, E., Smith, C. J., Vasilev, C. A., \& Stevens, A. L. (2008). Parent-child interactions, peripheral serotonin, and self-inflicted injury in adolescents. Journal of Consulting and Clinical Psychology, 76, 15-21. http://dx.doi.org/10.1037/0022006X.76.1.15

Cuthbert, B. N., \& Insel, T. R. (2013). Toward the future of psychiatric diagnosis: The seven pillars of RDoC. BMC Medicine, 11, 126. http:// dx.doi.org/10.1186/1741-7015-11-126

Cuthbert, B. N., \& Kozak, M. J. (2013). Constructing constructs for psychopathology: The NIMH research domain criteria. Journal of Abnormal Psychology, 122, 928-937. http://dx.doi.org/10.1037/a0034028

Daros, A. R., Zakzanis, K. K., \& Ruocco, A. C. (2013). Facial emotion recognition in borderline personality disorder. Psychological Medicine, 43, 1953-1963. http://dx.doi.org/10.1017/S0033291712002607

Domes, G., Schulze, L., \& Herpertz, S. C. (2009). Emotion recognition in borderline personality disorder-a review of the literature. Journal of Personality Disorders, 23, 6-19. http://dx.doi.org/10.1521/pedi.2009.23 .1.6

Donegan, N. H., Sanislow, C. A., Blumberg, H. P., Fulbright, R. K., Lacadie, C., Skudlarski, P., .. . Wexler, B. E. (2003). Amygdala hyperreactivity in borderline personality disorder: Implications for emotional dysregulation. Biological Psychiatry, 54, 1284-1293. http://dx .doi.org/10.1016/S0006-3223(03)00636-X

Ebner-Priemer, U. W., Badeck, S., Beckmann, C., Wagner, A., Feige, B., Weiss, I., . . Bohus, M. (2005). Affective dysregulation and dissociative experience in female patients with borderline personality disorder: A 
startle response study. Journal of Psychiatric Research, 39, 85-92. http://dx.doi.org/10.1016/j.jpsychires.2004.05.001

Ebner-Priemer, U. W., Eid, M., Kleindienst, N., Stabenow, S., \& Trull, T. J. (2009). Analytic strategies for understanding affective (in)stability and other dynamic processes in psychopathology. Journal of Abnormal Psychology, 118, 195-202. http://dx.doi.org/10.1037/a0014868

Ebner-Priemer, U. W., Kuo, J., Kleindienst, N., Welch, S. S., Reisch, T., Reinhard, I., . . . Bohus, M. (2007a). State affective instability in borderline personality disorder assessed by ambulatory monitoring. Psychological Medicine, 37, 961-970. http://dx.doi.org/10.1017/ S0033291706009706

Ebner-Priemer, U. W., Kuo, J., Welch, S. S., Thielgen, T., Witte, S., Bohus, M., \& Linehan, M. M. (2006). A valence-dependent groupspecific recall bias of retrospective self-reports: A study of borderline personality disorder in everyday life. Journal of Nervous and Mental Disease, 194, 774-779. http://dx.doi.org/10.1097/01.nmd.0000239900 .46595 .72

Ebner-Priemer, U. W., \& Sawitzki, G. (2007). Ambulatory assessment of affective instability in borderline personality disorder-the effect of the sampling frequency. European Journal of Psychological Assessment, 23, 238-247. http://dx.doi.org/10.1027/1015-5759.23.4.238

Ebner-Priemer, U. W., \& Trull, T. J. (2009). Ecological momentary assessment of mood disorders and mood dysregulation. Psychological Assessment, 21, 463-475. http://dx.doi.org/10.1037/a0017075

Ebner-Priemer, U. W., Welch, S. S., Grossman, P., Reisch, T., Linehan, M. M., \& Bohus, M. (2007b). Psychophysiological ambulatory assessment of affective dysregulation in borderline personality disorder. Psychiatry Research, 150, 265-275. http://dx.doi.org/10.1016/j.psychres .2006 .04 .014

Elices, M., Soler, J., Fernández, C., Martín-Blanco, A., Portella, M. J., Pérez, V., . . C Carlos Pascual, J. (2012). Physiological and self-assessed emotional responses to emotion-eliciting films in borderline personality disorder. Psychiatry Research, 200(2-3), 437-443. http://dx.doi.org/ 10.1016/j.psychres.2012.07.020

Fahrenberg, J., Huettner, P., \& Leonhart, R. (2001). MONITOR: Acquisition of psychological data by a hand-held PC. In J. Fahrenberg \& M. Myrtek (Eds.), Progress in ambulatory assessment: Computer-assisted psychological and psychophysiological methods in monitoring and field studies (pp. 93-112). Seattle: Hogrefe \& Huber.

First, M. B., Spitzer, R. L., Gibbon, M., Williams, J. B. W., \& Benjamin, L. S. (1997). User's guide for the structured clinical interview for DSM-IV Axis I disorders (SCID-I): Clinical version. Washington: American Psychiatric Press.

Fischer, A. H. (Ed.). (2000). Gender and emotion. New York: Cambridge University Press. http://dx.doi.org/10.1017/CBO9780511628191

Fydrich, T., Renneberg, B., Schmitz, B., \& Wittchen, H. U. (1997). SKID-II. Strukturiertes klinisches Interview für DSM-IV. Achse II: Persönlichkeitsstörungen. Göttingen: Hogrefe.

Gratz, K. L., Rosenthal, M. Z., Tull, M. T., Lejuez, C. W., \& Gunderson, J. G. (2010). An experimental investigation of emotional reactivity and delayed emotional recovery in borderline personality disorder: The role of shame. Comprehensive Psychiatry, 51, 275-285. http://dx.doi.org/ 10.1016/j.comppsych.2009.08.005

Hazlett, E. A., Speiser, L. J., Goodman, M., Roy, M., Carrizal, M., Wynn, J. K., . . . New, A. S. (2007). Exaggerated affect-modulated startle during unpleasant stimuli in borderline personality disorder. Biological Psychiatry, 62, 250-255. http://dx.doi.org/10.1016/j.biopsych.2006.10.028

Herpertz, S. C., Dietrich, T. M., Wenning, B., Krings, T., Erberich, S. G., Willmes, K., . . Sass, H. (2001). Evidence of abnormal amygdala functioning in borderline personality disorder: A functional MRI study. Biological Psychiatry, 50, 292-298. http://dx.doi.org/10.1016/S00063223(01)01075-7

Herpertz, S. C., Kunert, H. J., Schwenger, U. B., \& Sass, H. (1999). Affective responsiveness in borderline personality disorder: A psycho- physiological approach. The American Journal of Psychiatry, 156, $1550-1556$.

Jacob, G. A., Hellstern, K., Ower, N., Pillmann, M., Scheel, C. N., Rüsch, N., \& Lieb, K. (2009). Emotional reactions to standardized stimuli in women with borderline personality disorder: Stronger negative affect, but no differences in reactivity. Journal of Nervous and Mental Disease, 197, 808-815. http://dx.doi.org/10.1097/NMD.0b013e3181bea44d

Koenigsberg, H. W., Fan, J., Ochsner, K. N., Liu, X., Guise, K. G., Pizzarello, S., . . Siever, L. J. (2009). Neural correlates of the use of psychological distancing to regulate responses to negative social cues: A study of patients with borderline personality disorder. Biological Psychiatry, 66, 854-863. http://dx.doi.org/10.1016/j.biopsych.2009.06.010

Kuo, J. R., \& Linehan, M. M. (2009). Disentangling emotion processes in borderline personality disorder: Physiological and self-reported assessment of biological vulnerability, baseline intensity, and reactivity to emotionally evocative stimuli. Journal of Abnormal Psychology, 118, 531-544. http://dx.doi.org/10.1037/a0016392

Kuppens, P., Allen, N. B., \& Sheeber, L. B. (2010). Emotional inertia and psychological maladjustment. Psychological Science, 21, 984-991. http://dx.doi.org/10.1177/0956797610372634

Kuppens, P., Oravecz, Z., \& Tuerlinckx, F. (2010). Feelings change: Accounting for individual differences in the temporal dynamics of affect. Journal of Personality and Social Psychology, 99, 1042-1060. http://dx.doi.org/10.1037/a0020962

Lieb, K., Zanarini, M. C., Schmahl, C., Linehan, M. M., \& Bohus, M. (2004). Borderline personality disorder. The Lancet, 364, 453-461. http://dx.doi.org/10.1016/S0140-6736(04)16770-6

Limberg, A., Barnow, S., Freyberger, H. J., \& Hamm, A. O. (2011) Emotional vulnerability in borderline personality disorder is cue specific and modulated by traumatization. Biological Psychiatry, 69, 574-582. http://dx.doi.org/10.1016/j.biopsych.2010.10.024

Linehan, M. M. (1993). Cognitive-behavioral treatment of borderline personality disorder. New York, NY: Guilford Press.

Links, P. S., Eynan, R., Heisel, M. J., Barr, A., Korzekwa, M., McMain, S., \& Ball, J. S. (2007). Affective instability and suicidal ideation and behavior in patients with borderline personality disorder. Journal of Personality Disorders, 21, 72-86. http://dx.doi.org/10.1521/pedi.2007 21.1.72

Loranger, A. W. (1999). International Personality Disorder Examination (IPDE): DSM-IV and ICD-10 modules. Odessa: Psychological Assessment Resources.

Matzke, B., Herpertz, S. C., Berger, C., Fleischer, M., \& Domes, G. (2014) Facial reactions during emotion recognition in borderline personality disorder: A facial electromyography study. Psychopathology. Advance online publication.

Minzenberg, M. J., Fan, J., New, A. S., Tang, C. Y., \& Siever, L. J. (2007) Fronto-limbic dysfunction in response to facial emotion in borderline personality disorder: An event-related fMRI study. Psychiatry Research Neuroimagin, 155, 231-243. http://dx.doi.org/10.1016/j.pscychresns 2007.03.006

Nica, E. I., \& Links, P. S. (2009). Affective instability in borderline personality disorder: Experience sampling findings. Current Psychiatry Reports, 11, 74-81. http://dx.doi.org/10.1007/s11920-009-0012-2

Oravecz, Z., Tuerlinckx, F., \& Vandekerckhove, J. (2011). A hierarchical latent stochastic differential equation model for affective dynamics. Psychological Methods, 16, 468-490. http://dx.doi.org/10.1037/ a0024375

Oravecz, Z., Tuerlinckx, F., \& Vandekerckhove, J. (2012). BHOUM: Bayesian Hierarchial Ornstein-Uhlenbeck Modeling [Computer Software]. Retrieved from http://www.zitaoravecz.net/

Raudenbush, S. W., Bryk, A. S., \& Congdon, R. (2004). HLM 6 for Windows [Computer software]. Skokie, IL: Scientific Software International, Inc. 
Reisch, T., Ebner-Priemer, U. W., Tschacher, W., Bohus, M., \& Linehan, M. M. (2008). Sequences of emotions in patients with borderline personality disorder. Acta Psychiatrica Scandinavica, 118, 42-48. http:// dx.doi.org/10.1111/j.1600-0447.2008.01222.x

Reitz, S., Krause-Utz, A., Pogatzki-Zahn, E. M., Ebner-Priemer, U., Bohus, M., \& Schmahl, C. (2012). Stress regulation and incision in borderline personality disorder-a pilot study modeling cutting behavior. Journal of Personality Disorders, 26, 605-615. Advance online publication. http://dx.doi.org/10.1521/pedi.2012.26.4.605

Rosenthal, M. Z., Gratz, K. L., Kosson, D. S., Cheavens, J. S., Lejuez, C. W., \& Lynch, T. R. (2008). Borderline personality disorder and emotional responding: A review of the research literature. Clinical Psychology Review, 28, 75-91. http://dx.doi.org/10.1016/j.cpr.2007.04 .001

Russell, J. A. (2003). Core affect and the psychological construction of emotion. Psychological Review, 110, 145-172. http://dx.doi.org/10.1037/ 0033-295X.110.1.145

Sadikaj, G., Moskowitz, D. S., Russell, J. J., Zuroff, D. C., \& Paris, J. (2013). Quarrelsome behavior in borderline personality disorder: Influence of behavioral and affective reactivity to perceptions of others. Journal of Abnormal Psychology, 122, 195-207. http://dx.doi.org/10.1037/ a0030871

Sadikaj, G., Russell, J. J., Moskowitz, D. S., \& Paris, J. (2010). Affect dysregulation in individuals with borderline personality disorder: Persistence and interpersonal triggers. Journal of Personality Assessment, 92, 490-500. http://dx.doi.org/10.1080/00223891.2010.513287

Santangelo, P., Bohus, M., \& Ebner-Priemer, U. W. (2014). Ecological momentary assessment in borderline personality disorder: A review of recent findings and methodological challenges. Journal of Personality Disorders, 28, 555-576.

Santangelo, P., Reinhard, I., Mussgay, L., Steil, R., Sawitzki, G., Klein, C., . . Ebner-Priemer, U. W. (2014). Specificity of affective instability in patients with borderline personality disorder compared to posttraumatic stress disorder, bulimia nervosa, and healthy controls. Journal of Abnormal Psychology, 123, 258-272. http://dx.doi.org/10.1037/a0035619

Schmahl, C. G., Elzinga, B. M., Ebner, U. W., Simms, T., Sanislow, C., Vermetten, E., . . . Bremner, J. D. (2004). Psychophysiological reactivity to traumatic and abandonment scripts in borderline personality and posttraumatic stress disorders: A preliminary report. Psychiatry Research, 126, 33-42. http://dx.doi.org/10.1016/j.psychres.2004.01.005

Siever, L. J., Torgersen, S., Gunderson, J. G., Livesley, W. J., \& Kendler, K. S. (2002). The borderline diagnosis III: Identifying endophenotypes for genetic studies. Biological Psychiatry, 51, 964-968. http://dx.doi org/10.1016/S0006-3223(02)01326-4

Skodol, A. E., Gunderson, J. G., Pfohl, B., Widiger, T. A., Livesley, W. J., \& Siever, L. J. (2002a). The borderline diagnosis I: Psychopathology, comorbidity, and personality structure. Biological Psychiatry, 51, 936950. http://dx.doi.org/10.1016/S0006-3223(02)01324-0

Skodol, A. E., Siever, L. J., Livesley, W. J., Gunderson, J. G., Pfohl, B., \& Widiger, T. A. (2002b). The borderline diagnosis II: Biology, genetics, and clinical course. Biological Psychiatry, 51, 951-963. http://dx.doi .org/10.1016/S0006-3223(02)01325-2

Staebler, K., Gebhard, R., Barnett, W., \& Renneberg, B. (2009). Emotional responses in borderline personality disorder and depression: Assessment during an acute crisis and 8 months later. Journal of Behavior Therapy and Experimental Psychiatry, 40, 85-97. http://dx.doi.org/10.1016/j .jbtep.2008.04.003

Stiglmayr, C. E., Grathwol, T., Linehan, M. M., Ihorst, G., Fahrenberg, J., \& Bohus, M. (2005). Aversive tension in patients with borderline personality disorder: A computer-based controlled field study. Acta Psychiatrica Scandinavica, 111, 372-379. http://dx.doi.org/10.1111/j 1600-0447.2004.00466.x

Trull, T. J., \& Ebner-Priemer, U. (2013). Ambulatory assessment. Annual Review of Clinical Psychology, 9, 151-176. http://dx.doi.org/10.1146/ annurev-clinpsy-050212-185510

Trull, T. J., Solhan, M. B., Tragesser, S. L., Jahng, S., Wood, P. K., Piasecki, T. M., \& Watson, D. (2008). Affective instability: Measuring a core feature of borderline personality disorder with ecological momentary assessment. Journal of Abnormal Psychology, 117, 647-661. http:// dx.doi.org/10.1037/a0012532

Wang, L. P., Hamaker, E., \& Bergeman, C. S. (2012). Investigating inter-individual differences in short-term intra-individual variability. Psychological Methods, 17, 567-581. http://dx.doi.org/10.1037/a0029317

Wilhelm, P., \& Schoebi, D. (2007). Assessing mood in daily life-structural validity, sensitivity to change, and reliability of a short-scale to measure three basic dimensions of mood. European Journal of Psychological Assessment, 23, 258-267. http://dx.doi.org/10.1027/1015-5759 .23 .4 .258

Wittchen, H. U., Wunderlich, U., \& Gruschwitz, S. (1997). SKID. Strukturiertes Klinisches Interview für DSM-IV Achse I. Göttingen: Hogrefe.

Received January 24, 2014

Revision received October 7, 2014

Accepted October 11, 2014 